\title{
REMARKS ON THE NEF CONE ON SYMMETRIC PRODUCTS OF CURVES
}

\author{
F. BASTIANELLI
}

\begin{abstract}
Let $C$ be a very general curve of genus $g$ and let $C^{(2)}$ be its second symmetric product. This paper concerns the problem of describing the convex cone $N$ ef $\left(C^{(2)}\right)_{\mathbb{R}}$ of all numerically effective $\mathbb{R}$-divisors classes in the Néron-Severi space $N^{1}\left(C^{(2)}\right) \mathbb{R}$. In a recent work, Julius Ross improved the bound on $N$ ef $\left(C^{(2)}\right)_{\mathbb{R}}$ in the case of genus five. By using his techniques and by studying the gonality of the curves lying on $C^{(2)}$, we give new bounds on the nef cone of $C^{(2)}$ when $C$ is a very general curve of genus $5 \leq g \leq 8$.
\end{abstract}

\section{INTRODUCTION}

Let $C$ be a smooth irreducible complex projective curve of genus $g \geq 0$. Denote by $C^{(2)}$ the second symmetric product of $C$ which is the smooth surface parametrizing the unordered pairs of point of $C$. On $C^{(2)}$, we can define some divisors in a natural way as follow: fixing a point $p \in C$ there are the divisor $X_{p}:=\{p+q \mid q \in C\}$ and the diagonal divisor $\Delta:=\{q+q \mid q \in C\}$. Let $x_{p}$ and $\delta$ denote the classes of such divisors in the Néron-Severi group $N^{1}\left(C^{(2)}\right)$. Since the class $x_{p}$ of the divisor $X_{p}$ is independent from the choice of the point $p \in C$, we simply denote by $x$ such class.

Let $N$ ef $\left(C^{(2)}\right)_{\mathbb{R}}$ be the convex cone of all numerically effective $\mathbb{R}$-divisors classes on $C^{(2)}$ and consider the plane $\Pi \subset N^{1}\left(C^{(2)}\right)_{\mathbb{R}}$ spanned by $x$ and $\delta$. Our aim is to study the two-dimensional subcone $N$ obtained as intersection of the nef cone with the plane $\Pi$. This is equivalent to determine the two boundary rays of $N$. The first one is the dual ray of the diagonal divisor class via the intersection pairing. Namely, since the diagonal is an irreducible curve of negative self intersection, it spans a boundary ray of the effective cone of curves, thus one boundary of the ample cone is $\left\{\alpha \in N^{1}\left(C^{(2)}\right) \mid(\delta \cdot \alpha)=0\right\}$. The other ray is determined by the real number

$$
\tau(C)=\inf \left\{t>0 \mid(t+1) x-\frac{\delta}{2} \text { is ample }\right\} .
$$

Hence the problem of describing the cone $N$ is equivalent to compute $\tau(C)$. Notice that if $(t+1) x-\frac{\delta}{2}$ is an ample class of $N^{1}\left(C^{(2)}\right)_{\mathbb{R}}$, then it must have positive self intersection and hence $\tau(C) \geq \sqrt{g}$.

We note that when $C$ is a genus $g$ curve with very general moduli (i.e. there exists a countable collection of proper subvarieties of the moduli space $\mathcal{M}_{g}$ such that the corresponding point $[C]$ in $\mathcal{M}_{g}$ is not contained in the union of those subvarieties) the vector

This work has been partially supported by 1) PRIN 2007 "Spazi di moduli e teorie di Lie"; 2) Indam (GNSAGA); 3) FAR 2008 (PV) "Varietá algebriche, calcolo algebrico, grafi orientati e topologici". 
space $N^{1}\left(C^{(2)}\right)_{\mathbb{R}}$ is spanned by $x$ and $\delta / 2$, hence $N$ is the whole nef cone (cf. [1, Chapter VIII Section 5]).

When $C$ is a very general curve of genus $g \leq 3$, the problem of describing the cone $N$ is totally understood (for details see e.g. [5] and [2]).

There is an important conjecture - due to Alexis Kouvidakis - which asserts that if $C$ is a very general curve of genus $g \geq 4$, then $\tau(C)=\sqrt{g}$, i.e. the nef cone is as large as possible. In [5], the statement has been proved when $g$ is a perfect square. Moreover Kouvidakis proved that

$$
\sqrt{g} \leq \tau(C) \leq \frac{g}{[\sqrt{g}]}
$$

for any very general curve of genus $g \geq 5$. The cases $g=5$ and $g \geq 10$ have been recently improved.

In particular, by using a bound on the Seshadri constant at $g$ general points of $\mathbb{P}^{2}$ (see [9]), as a consequence of a result due to Ciliberto and Kouvidakis (cf. [2] and [8, Corollary 1.7]), we have that

$$
\tau(C) \leq \frac{\sqrt{g}}{\sqrt{1-\frac{1}{8 g}}}
$$

for any very general curve of genus $g \geq 10$. Furthermore, when $C$ is a genus five curve with very general moduli, Julius Ross proved that $\tau(C) \leq 16 / 7$ (cf. [8, Section 4]).

This paper concerns mainly the description of the nef cone of $C^{(2)}$ when $C$ is a very general curve of low genus. In particular, we prove the following:

Theorem 1.1. Consider the rational numbers

$$
\tau_{5}=\frac{9}{4}, \quad \tau_{6}=\frac{37}{15}, \quad \tau_{7}=\frac{189}{71} \quad \text { and } \quad \tau_{8}=\frac{54}{19} .
$$

Let $C$ be a smooth irreducible complex projective curve of genus $5 \leq g \leq 8$ and assume that $C$ has very general moduli. Then

$$
\tau(C) \leq \tau_{g}
$$

Notice that $\tau_{5}<\frac{16}{7}$ and that $\tau_{g}<\frac{g}{[\sqrt{g}]}$ for $g=6,7,8$. Thus Theorem 1.1 gives a slight improvement of the bounds on the ample cone of $C^{(2)}$.

The argument of the proof is based on the main theorem in [8] together with the techniques used by Ross, due to Ein and Lazarsfeld (see [3]). Moreover, to be able to deduce the bounds in the statement of Theorem 1.1, we present two other results. The first one is a slight refinement of [3, Corollary 1.2] and the second one is an extension of a result of Pirola about curves on very general abelian varieties of dimension grater than 2 (cf. [7]). In particular, we prove that the Jacobian variety $J(C)$ of a very general curve $C$ of genus $g \geq 3$ does not contain hyperelliptic curves (see Proposition 2.3). 


\section{Preliminaries}

In the following, we work over the field of complex numbers. We say that a point on a complex projective variety $x \in X$ is very general if there exists a countable collection of proper subvarieties of $X$ such that $x$ is not contained in the union of those subvarieties. Then a curve $C$ of genus $g$ is said to be very general if it is smooth and its corresponding point in the moduli space $\mathcal{M}_{g}$ is very general.

2.1. Divisors on $C^{(2)}$. Let $C$ be a smooth irreducible complex projective curve of genus $g \geq 1$. Its second symmetric product is defined as the quotient of the ordinary product $C \times C$ by the natural involution. Hence the quotient map $\pi: C \times C \longrightarrow C^{(2)}$ is defined by $\pi\left(p_{1}, p_{2}\right)=p_{1}+p_{2}$ for $p_{1}, p_{2} \in C$ and it is ramified along the diagonal. Let $N^{1}\left(C^{(2)}\right)_{\mathbb{R}}$ be the vector space of the numerical equivalence class of $\mathbb{R}$-divisors and consider the classes $x, \delta \in N^{1}\left(C^{(2)}\right)_{\mathbb{R}}$ defined in the introduction. As the diagonal divisor on $C \times C$ defines a line bundle invariant under the natural involution, it induces a line bundle on $C^{(2)}$. Since the natural map $\pi$ ramifies along the diagonal $\Delta \subset C^{(2)}$, the square of the latter line bundle is isomorphic to the one induced by $\Delta$ on $C^{(2)}$ and its numerical equivalence class is $\frac{\delta}{2}$.

We assume hereafter that $C$ is a very general curve. Hence the classes $x$ and $\frac{\delta}{2}$ span the whole $N^{1}\left(C^{(2)}\right)_{\mathbb{R}}$. The intersection numbers between these numerical classes are $\left(x^{2}\right)=1$, $\left(\left(\frac{\delta}{2}\right)^{2}\right)=1-g,\left(x \cdot \frac{\delta}{2}\right)=1$ and the intersection of divisor classes spanned by $x$ and $\frac{\delta}{2}$ is governed by the following formula:

$$
\left((a+b) x-b \frac{\delta}{2}\right) \cdot\left((m+n) x-n \frac{\delta}{2}\right)=a m-b n g .
$$

2.2. Seshadri constants. Let $Y$ be a smooth complex projective variety and let $L \in$ $N^{1}(Y)_{\mathbb{R}}$ be a nef class. Then we define the Seshadri constant of $L$ at a point $y \in Y$ to be the real number

$$
\epsilon(y ; Y, L):=\inf _{E} \frac{(L \cdot E)}{\text { mult }_{y} E},
$$

where the infimum is taken over the irreducible curves $E$ passing through $y$.

Then let us state the main theorem in [8] connecting Seshadri constants on the second symmetric product of a curve of genus $g-1$ and the ample cone of the second symmetric product of a very general curve of genus $g$.

Theorem 2.1 (Ross). Let $D$ be a smooth curve of genus $g-1$. Let $a, b$ be two positive real numbers such that $a / b \geq \tau(D)$ and for a very general point $y \in D^{(2)}$

$$
\epsilon\left(y ; D^{(2)},(a+b) x-b \frac{\delta}{2}\right) \geq b .
$$

Then for a very general curve $C$ of genus $g$,

$$
\tau(C) \leq \frac{a}{b}
$$


As we anticipated in the introduction, Ross applies the theorem above to the computation of a bound for the ample cone on the second symmetric product of a very general curve of genus five (see [8, Section 4]). One important tool involved in the proof is Corollary 1.2 in [3].

The following lemma is a slight improvement of the latter result - under some additional hypothesis - and the proof follows the same argument. For a curve $E$, we denote by $\widetilde{E}$ its normalization and by $\operatorname{gon}(\widetilde{E})$ the gonality of the curve $\widetilde{E}$. Moreover, we define the gonality of $E$ as the gonality of its normalization.

Lemma 2.2. Let $Y$ be a smooth complex projective surface. Let $T$ be a smooth variety and consider a family $\left\{y_{t} \in E_{t}\right\}_{t \in T}$ consisting of a curve $E_{t} \subset Y$ through a very general point $y_{t} \in X$ such that mult $y_{t} E_{t} \geq m$ for any $t \in T$ and for some $m \geq 2$.

If the central fibre $E_{0}$ is a reduced irreducible curve and the family is non-trivial, then

$$
E_{0}^{2} \geq m(m-1)+\operatorname{gon}\left(\widetilde{E}_{0}\right) .
$$

Proof. As in [3], let us consider the blowing-up $f: Y^{\prime} \longrightarrow Y$ of $Y$ at $y_{0}$ and let $F \subset Y^{\prime}$ be the exceptional divisor. Let $E_{0}^{\prime}$ be the strict transform of $E_{0}$. Then $E_{0}^{\prime}=f^{*} E_{0}-k F$ with $k=$ mult $_{y_{0}} E_{0} \geq m$ and hence $E_{0}^{\prime}$ is the blowing-up of $E_{0}$ at $y_{0}$.

Since each $y_{t}$ is a singular point of $E_{t}$, the variety $T$ parametrizing the family must be at least two-dimensional. Then, up to consider a subfamily, we assume that the dimension of $T$ is 2 . Let $\left(t_{1}, t_{2}\right) \in \mathbb{C}^{2}$ be the local coordinates of $T$ around $t=0$. Consider the sections $s_{1}=\rho\left(\frac{d}{d t_{1}}\right), s_{2}=\rho\left(\frac{d}{d t_{2}}\right) \in H^{0}\left(E_{0}, \mathcal{O}_{E_{0}}\left(E_{0}\right)\right)$ of the normal bundle to $C$ in $Y$, where $\rho$ is the Kodaira-Spencer deformation map. Thus, by [3, Lemma 1.1] and being the family non-trivial, $s_{1}$ and $s_{2}$ induce two non-zero sections $s_{1}^{\prime}, s_{2}^{\prime} \in H^{0}\left(E_{0}^{\prime}, f^{*}\left(\mathcal{O}_{E_{0}}\left(E_{0}\right)\right) \otimes \mathcal{O}_{Y^{\prime}}((1-\right.$ $\left.m) F)\left.\right|_{E_{0}^{\prime}}\right)$. By last two sections we define a map $\phi: E_{0}^{\prime} \longrightarrow \mathbb{P}^{1}$ which extends to a map $\widetilde{\phi}: \widetilde{E}_{0} \longrightarrow \mathbb{P}^{1}$, hence

$E_{0}^{2}=\operatorname{deg} \mathcal{O}_{E_{0}}\left(E_{0}\right)=\left.\operatorname{deg} f^{*}\left(\mathcal{O}_{E_{0}}\left(E_{0}\right)\right)\right|_{E_{0}^{\prime}} \geq(m-1)\left(F \cdot E_{0}^{\prime}\right)+\operatorname{deg} \phi \geq m(m-1)+g o n\left(\tilde{E}_{0}\right)$

and this concludes the proof.

2.3. Gonality of curves on $C^{(2)}$. Let $C$ be a very general curve of genus $g \geq 3$. Our next task is to study the gonality of the curves lying on the second symmetric product $C^{(2)}$, so that we can combine this study with the previous lemma.

As $C$ is assumed to be very general and its genus is greater than two, we have that $C$ is non-hyperelliptic and the second symmetric product $C^{(2)}$ embeds into the Jacobian variety $J(C)$ via the Abel map. Then, let us focus on the gonality of curves lying on $J(C)$.

To start we recall that any Abelian variety does not contain rational curves. Indeed, if $R$ were a rational curve contained in an Abelian variety $A$, then the inclusion map should factor through the Jacobian variety of $R$. As the Jacobian variety of a rational curve is a point, we get a contradiction. 
In [7], Gian Pietro Pirola proves that the generic Abelian variety of dimension greater than 2 does not contain hyperelliptic curve of any genus, where elliptic curves are considered as special cases of hyperelliptic curves. Since for any 3-dimensional Abelian variety there exists an isogeny to a Jacobian variety of a genus three curve, we deduce that for any very general curve $C$ of genus three, its Jacobian variety $J(C)$ does not contain hyperelliptic curves. Thus by using a degeneration argument we have the following.

Proposition 2.3. If $C$ is a very general curve of genus $g \geq 3$, the Jacobian variety $J(C)$ does not contain hyperelliptic curves.

Proof. As we said above, the case of genus 3 is a consequence of [7, Theorem 2]. Then by induction on the genus, suppose that the statement holds for every very general curve of genus $g-1$.

So, consider a very general curve $D$ of genus $g-1$ and a smooth elliptic curve $E$, together with two points $p \in D$ and $q \in E$. Let $C_{0}$ be the nodal curve obtained by gluing $D$ and $E$ at $p$ and $q$. Let $\mathcal{C} \longrightarrow \Delta$ be a proper flat family over a disc $\Delta$ such that the fiber over $0 \in \Delta$ is $C_{0}$ and for any $t \neq 0$ the fiber $C_{t}$ is a smooth curve of genus $g$.

Then consider the Jacobian bundle over $\Delta$ of $\mathcal{C}$, that is $J(\mathcal{C}) \longrightarrow \Delta$ with $J(\mathcal{C})_{t}=J\left(C_{t}\right)$ for all $t \in \Delta-\{0\}$. By contradiction, assume that the fiber $J\left(C_{t}\right)$ of $J(\mathcal{C})$ contains an hyperelliptic curve $X_{t}$ for very general $t \in \Delta-\{0\}$. Hence - up to restrict the disk $\Delta$ we can define the following map of families over the punctured disk $\Delta-\{0\}$

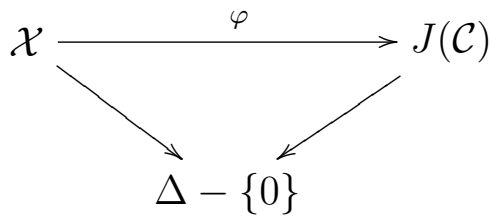

where $\varphi_{t}: X_{t} \hookrightarrow J\left(C_{t}\right)$ is the inclusion map.

We have $J(\mathcal{C})_{0}=J(D) \times J(E)=J(D) \times E$. Denote by $\pi_{1}: J(D) \times E \longrightarrow J(D)$ the natural projection map on the first factor. Let $X_{0} \subset J(D) \times E$ be the flat limit of the family of hyperelliptic curves $\mathcal{X}$ at $t=0$. Since the very general fiber $X_{t}$ generates $J\left(C_{t}\right)$ as a group, then $X_{0}$ must generate $J(D) \times E$. Thus $\pi_{1}\left(X_{0}\right) \subset J(D)$ cannot be 0-dimensional and hence it is a non-rational curve on $J(D)$. Then $X_{0}$ has some non-rational irreducible components that are all hyperelliptic curves. Therefore all the irreducible components of $\pi_{1}\left(X_{0}\right)$ are hyperelliptic and we have a contradiction because $D$ has genus $g-1$ and its Jacobian variety $J(D)$ does not contain hyperelliptic cuves by induction.

As a consequence of the proposition, the following holds.

Corollary 2.4. Let $C$ be a very general curve of genus $g \geq 3$. Then there are neither rational curves nor hyperelliptic curves lying on $C^{(2)}$. 


\section{Proof of Theorem 1.1}

This section is devoted to prove Theorem 1.1. To start we focus on the case of genus five. We follow the argument of J. Ross in [8, Section 4] and we are proving that for any very general curve $C$ of genus five we have

$$
\tau(C) \leq \frac{9}{4} .
$$

So, let $D$ be a very general curve of genus 4 and let $D^{(2)}$ be its second symmetric product. Then set $a=9, b=4$ and consider the numerical equivalence class

$$
L:=(a+b) x-b \frac{\delta}{2} \in N^{1}\left(D^{(2)}\right) .
$$

Since $\tau(D)=2$, by Theorem 2.1 we deduce that to prove (3.1) it suffices to show that for a very general point $y \in D^{(2)}$

$$
\epsilon\left(y ; D^{(2)}, L\right) \geq b=4,
$$

i.e. there is not a reduced and irreducible curve $E$ passing through a general point $y \in D^{(2)}$, such that $(L \cdot E) /$ mult $_{y} E<b=4$.

Let us consider the set $\mathcal{F}$ of pairs $(F, z)$ such that $F \subset D^{(2)}$ is a reduced irreducible curve, $z \in F$ is a point and $(L \cdot F) /$ mult $_{z} F<4$. Since $\mathcal{F}$ consists of at most countably many algebraic families and the point $y \in D^{(2)}$ is assumed to be very general, the inequality (3.2) will be checked if each of these families is discrete.

Aiming for a contradiction, assume that there exists a family $\left\{y_{t} \in E_{t}\right\}_{t \in T}$ such that for all $t \in T$ the curve $E_{t} \subset D^{(2)}$ is reduced and irreducible, the point $y_{t} \in D^{(2)}$ is very general and

$$
\frac{\left(L \cdot E_{t}\right)}{\text { mult }_{y_{t}} E_{t}}<4 .
$$

As in [8], we note that for any reduced irreducible curve $E \subset D^{(2)}$ through a very general point $y \in D^{(2)}$ we have

$$
(L \cdot E) \geq b=4 .
$$

To see this fact, consider the numerical class $[E]=(n+\gamma) x-\gamma(\delta / 2) \in N^{1}\left(D^{(2)}\right)$. Since the class $x$ is ample, $(x \cdot E)=n>0$ and the claim is easily checked when $\gamma \leq 0$.

Then assume $\gamma>0$. Being $\tau(D)=2$, the diagonal is the only curve of $D^{(2)}$ with negative self intersection. Moreover, there exist at most finitely many irreducible curves of zero self intersection and numerical class $(n+\gamma) x-\gamma(\delta / 2)$, then we can assume that $E^{2}=n^{2}-4 \gamma^{2}>0$ as $y \in D^{(2)}$ is assumed to be very general. Hence $n \geq 2 \gamma+1$ and $(L \cdot E)=9 n-16 \gamma \geq 2 \gamma+9>4$ for all $\gamma>0$.

Thus by (3.3) and (3.4) we deduce that mult $_{y_{t}} E_{t}>\left(L \cdot E_{t}\right) / 4 \geq 1$ for any $t \in T$. Being $E_{t}$ reduced, for a general point $z \in E_{t}$ the multiplicity of $E_{t}$ at $z$ is one, therefore the family $\left\{y_{t} \in E_{t}\right\}_{t \in T}$ is non-trivial. 
Without loss of generality, let us assume that the central fibre $\left(E_{0}, y_{0}\right)$ is such that

$$
m:=\text { mult }_{y_{0}} E_{0} \leq \text { mult }_{y_{t}} E_{t}
$$

for any $t \in T$. Hence by Lemma 2.2 we have that the curve $E_{0}$ has self intersection $E_{0}^{2} \geq m(m-1)+\operatorname{gon}\left(\widetilde{E}_{0}\right)$, where $\widetilde{E}_{0}$ is the normalization of $E_{0}$.

Moreover, by Corollary 2.4 there are neither rational curves nor hyperelliptic curves lying on $D^{(2)}$. Therefore the gonality of $\widetilde{E}_{0}$ is at least three and

$$
E_{0}^{2} \geq m(m-1)+3
$$

Finally, by $(3.3)$ we deduce that $\left(L \cdot E_{0}\right) \leq 4 m-1$. Thus by Hodge Index Theorem we have

$$
m(m-1)+3 \leq E_{0}^{2} \leq \frac{\left(L \cdot E_{0}\right)^{2}}{L^{2}} \leq \frac{(4 m-1)^{2}}{17},
$$

but this is impossible. Hence we proved that if $C$ is a very general curve of genus $g=5$, then $\tau(C) \leq \frac{9}{4}$.

To conclude the proof of Theorem 1.1, we note that $\frac{9}{4}<\frac{37}{15}<\frac{189}{71}<\frac{54}{19}$. Hence it is still possible to apply Theorem 2.1 and - by using the very same argument - the proof for the cases $g=6,7,8$ is straightforward.

\section{ACKNOWLEDGEMENTS}

I am grateful to my Ph.D. advisor Gian Pietro Pirola for getting me interested in this problem and for the helpful suggestions to improve the results in Lemma 2.2 and Proposition 2.3.

\section{REFERENCES}

[1] E. Arbarello, M. Cornalba, P. A. Griffiths, J. Harris, Geometry of Algebraic Curves, Vol. I, Grundlehren der Mathematischen Wissenschaften [Fundamental Principles in Mathematical Sciences], 267, Springer-Verlag, New York, 1985.

[2] C. Ciliberto and A. Kouvidakis, On the symmetric product of a curve with general moduli, Geom. Dedicata, 78(3) (1999), 327-343.

[3] L. Ein and R. Lazarsfeld, Seshadri constants on smooth surfaces, Astrisque, 218 (1993), 177-186. Journes de Gomtrie Algbrique d'Orsay (Orsay, 1992).

[4] P. A. Griffiths, J. Harris, Principles of Algebraic Geometry, Pure and Applied Mathematics, Wiley Interscience, New York, 1978

[5] A. Kouvidakis, Divisors on symmetric products of curves, Trans. Amer. Math. Soc., 337(1) (1993), $117-128$.

[6] R. Lazarsfeld, Positivity in Algebraic Geometry, Vol. I, Ergebnisse der Mathematik und ihrer Grenzebiete (3), 48, Springer-Verlag, Berlin, 2004.

[7] G. P. Pirola, Curves on generic Kummer varieties, Duke Math. J., 59(3) (1989), 701-708.

[8] J. Ross, Seshadri constants on symmetric products of curves, Math. Res. Lett., 14(1) (2007), 63-75.

[9] B. Strycharz-Szemberg and T. Szemberg, Remarks on the Nagata conjecture, Serdica Math. J., 30(2-3) (2004), 405-430. 
Dipartimento di Matematica, via Ferrata 1, 27100 Pavia, Italy

E-mail address: francesco.bastianelli@unipv.it 